\title{
A Tale of Absent Crystalline Lens in an Eye with Retinoblastoma
}

\author{
Aditya Bansal Veena Noronha Subramanian Krishnakumar Vikas Khetan \\ Department of Vitreoretina and Oncology, Medical Research Foundation, Sankara Nethralaya, Chennai, India
}

\section{Established Facts}

- Invasion of the anterior segment by retinoblastoma is a well-recognized although uncommon complication.

- Invasion of lens capsule by a tumor has been rarely reported in humans.

\section{Novel Insights}

- We report a clinicopathologically proven absence of crystalline lens in an Asian child of Indian origin with retinoblastoma suggestive of lens involvement by tumor.

\section{Keywords}

Aphakia · Lens involvement by tumor · Retinoblastoma

\begin{abstract}
Purpose: To report the clinicopathological correlation of absent crystalline lens in an eye with retinoblastoma. Methods: A 1-year-old female child was brought to our patient services for pain and swelling in the right eye since the past few months. The child was diagnosed to have bilateral retinoblastoma and was suspected to have absence of crystalline lens in the right eye on the basis of clinical findings and ocular imaging. After 3 cycles of chemotherapy for suspected extraocular extension of tumor, she underwent enucleation. Absence of crystalline lens was confirmed on both gross and microscopic pathology. Results: We present a child with ret-
\end{abstract}

\section{KARGER}

(c) 2018 S. Karger AG, Basel

E-Mail karger@karger.com

www.karger.com/oop inoblastoma, suspected to have absence of crystalline lens clinically and on ocular imaging (ultrasound and MRI), which was finally confirmed on ocular pathology examination post enucleation. Conclusion: We report for the first time clinicopathologically proven absence of crystalline lens in an eye with retinoblastoma.

(c) 2018 S. Karger AG, Basel

\section{Introduction}

Retinoblastoma is a common intraocular malignancy in infancy or childhood, presenting with typical clinical features of leukocoria (white reflections in pupil) and strabismus, with an incidence rate of approximately 1 in 15,000-20,000 live births worldwide. Invasion of the an- 
Fig. 1. One-year-old female child presented with full chamber hyphema in the right eye (a) and retinoblastoma in the left eye (b). Ultrasonography revealed mass lesion with intraocular calcification and no definite lens echo in the right eye (c). MRI scan shows lobulated T2 hypointense intraocular mass lesion in both eyes. In the right eye, the lens was not visualized, whereas ocular coats were noted to be irregular without any obvious optic nerve extension, but a small bulge was noted temporal to the optic nerve head (d).

Fig. 2. After 3 cycles of neoadjuvant chemotherapy, the right eye showed resolution of hyphema with absence of iris tissue and crystalline lens (a), whereas the left eye revealed type 3 regression of retinoblastoma (b). Axial T2-weighted image of MRI shows phthisical right eye with disorganized intraocular soft tissue, small elevated lesion in the left eye, and no significant abnormality in brain parenchyma (c). The right eye underwent enucleation and at review after a month, a shell was fitted (d).
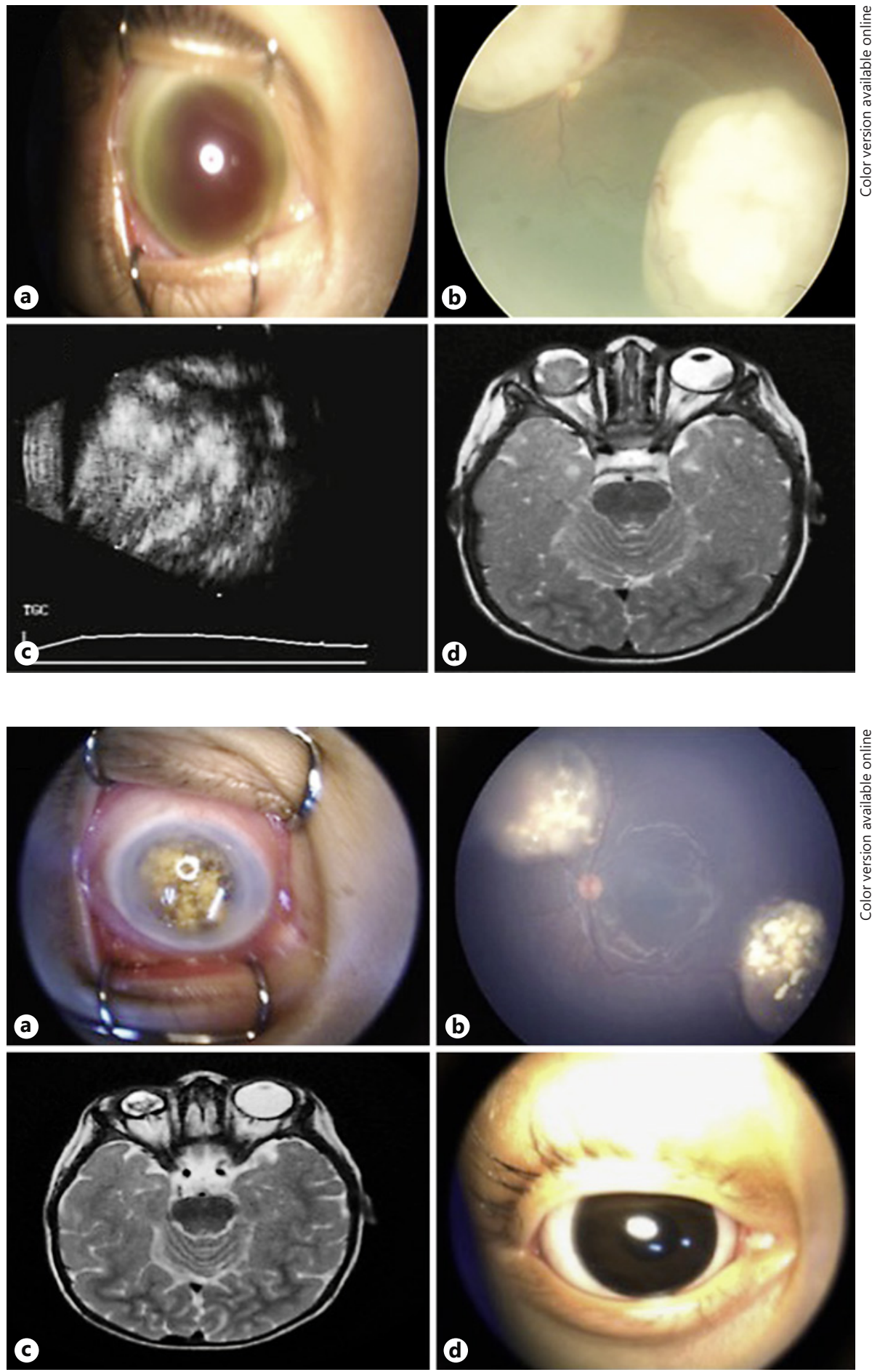

terior segment by retinoblastoma is a well-recognized although uncommon complication, and in that it may simulate ocular inflammation, which is a significant source of misdiagnosis [1]. Invasion of lens capsule by a tumor has been rarely reported in humans [2, 3]. Till date, only 1 case report by Gupta et al. [4] has reported lens invasion by ocular tumor in retinoblastoma. We report a case of an Asian child of Indian origin noted to have absence of crystalline lens in an eye with retinoblastoma. 

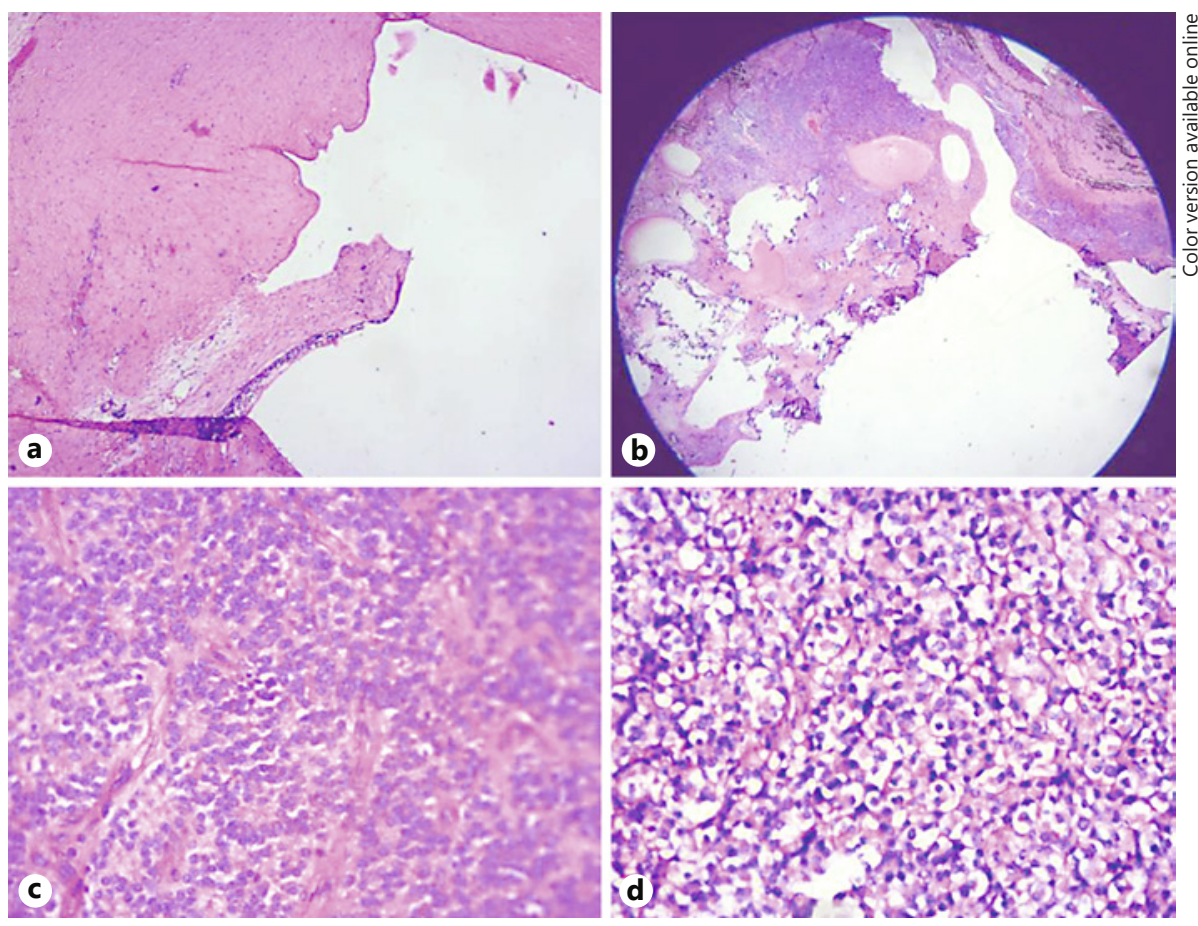

Fig. 3. Histopathological examination shows absence of crystalline lens in patellar fossa (a, b). Rosette pattern of tumor cells was noted (c). Tumor cells were noted invading the iris, ciliary body, and choroid.

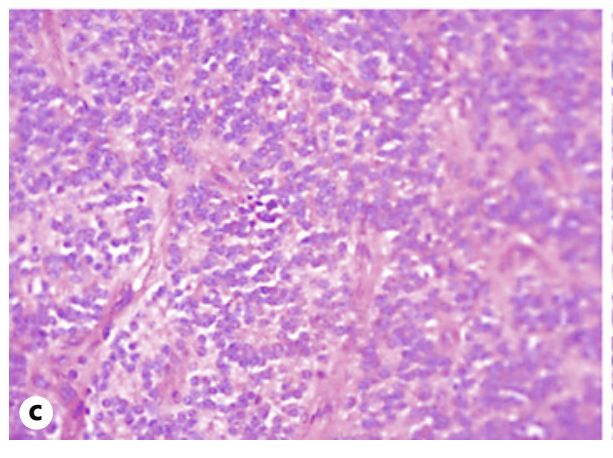

\section{Case Report}

A 1-year-old Asian female child of Indian origin, was brought to the outpatient services by her father with chief complaints of swelling of the right eye followed by development of white reflex over the past 9 months. There was no history of any ocular trauma, systemic ailments, or any ocular interventions. There was no family history of retinoblastoma. On examination, the child was noted to have a central, steady, and maintained fixation with the left eye only. Diffuse light examination of the right eye showed clear cornea with full chamber hyphema, i.e., grade 4 (Fig. 1a). Left-eye anterior segment was within normal limits and posterior segment was suspected to have retinoblastoma.

Ultrasonography for both eyes revealed a mass lesion with intraocular calcification with no definite lens echo in the right eye (Fig. 1c). Magnetic resonance imaging (MRI) showed right-eye enlarged globe with a lobulated T2 hypointense intraocular mass lesion $(19.3 \times 17.5 \mathrm{~mm})$. The lens was not visualized, whereas ocular coats were noted to be irregular without any obvious optic nerve extension but a small bulge was noted temporal to the optic nerve head (Fig. 1d). Similarly, the left eye revealed a domeshaped intraocular lesion posterior to the equator without any optic nerve or extraocular extension. Cerebrospinal fluid and bone marrow analysis were done to rule out systemic metastasis and were negative. Clinical findings were confirmed when the child was evaluated under general anesthesia. The child was diagnosed with bilateral retinoblastoma and as per International Classification of Retinoblastoma (ICRB) grouped as Group E in the right eye and Group $\mathrm{C}$ in the left eye.

The child underwent examination under anesthesia with neoadjuvant chemotherapy thrice at a 3 -week interval (as optic nerve head bulge was noted in the right eye on MRI). Meanwhile, hy- phema was noted to be cleared and clinically, absence of iris and lens was confirmed (Fig. 2a). MRI was repeated at the next followup, which revealed disorganized intraocular soft tissue and thickening of posterior ocular coat without any obvious enhancing intraocular mass lesion along with thinning of the optic nerve of the right eye (Fig. 2c). The left eye showed regressing of the temporal and superonasal mass lesion (Fig. 2b). She was advised to undergo regular examination under anesthesia with planning of right-eye enucleation and left-eye transpupillary thermotherapy. The child underwent the procedure as per plan followed by chemotherapy. The enucleated eye was sent for histopathological analysis.

Gross pathology revealed an intact phthisical globe with optic nerve measuring $15 \mathrm{~mm}$ in length and $3 \mathrm{~mm}$ in diameter. The globe was sectioned horizontally and cut section revealed clear cornea, rudimentary iris, absence of lens, and clear vitreous cavity; retina and optic nerve head could not be made out, choroid was of normal thickness, and sclera was thickened. Histopathological examination was made after cutting the callotes into bread loaf sections: pupil optic nerve axis (17 sections), lateral sections (8 sections), and separate section of the surgical end of the optic nerve ( 5 sections). Tumor cells were noted invading the iris, ciliary body, and choroid more than $3 \mathrm{~mm}$. It was noted to invade the pre-laminar portion of the optic nerve and anterior fibers of the sclera. The surgical end of the optic nerve was free from tumor cells (Fig. 3).

Absence of crystalline lens was noted clinically and confirmed on all ancillary tests and ocular pathology. Differentials were congenital aphakia (primary and secondary) or absorption of lens following tumor invasion. Primary congenital aphakia was ruled out as the rest of the ocular structure, especially the anterior segment, was within normal limits except for a rudimentary iris and 
there were no associated systemic features. Secondary congenital aphakia was ruled out as TORCH investigations were negative. The most probable diagnosis for the absence of the lens was involvement by tumor with consequent absorption of the lens and its capsule.

\section{Discussion}

The crystalline lens has long been regarded as tumorimmune tissue [5]. Even highly invasive tumors occupying the entire vitreous demonstrate well-defined borders at the lens capsule interface. Several hypotheses have been proposed for this. These include anaerobic metabolism of the lens, impermeability of the lens capsule, and genetic factors [5]. It has been speculated that chemicals like antiangiogenic factors, growth inhibitors, and anti-lens capsule digestion factors repel the tumor cells from the capsule. Besides, the lens capsule is the thickest basement membrane of the body and is thought to be impermeable to cells [6].

However, Gupta et al. [4] have reported invasion of lens capsule in a 2-year-old female. They reported that on gross pathology, the lens was displaced with the mass infiltrating the posterior and temporal equatorial surface of the lens. This was confirmed on histopathology, which revealed presence of the tumor cells in the lens matrix. In our case, we found absence of iris and crystalline lens from the day of presentation, which was also noted on ultrasonography and MRI. Histopathology confirmed the same. Congenital aphakia was ruled out considering the absence of other supportive findings. The most probable etiology for the absence of the lens and its capsule was tumor invasion with subsequent complete absorption.

In our case report, we followed IRSWG (International Retinoblastoma Study Working Group) guidelines of taking 25 sections of the globe for histopathological examination instead of serial sections; therefore, there may be a rare possibility of missing small remnant lens matter.

This report can be of value in understanding that lens involvement, though rare, can be possible in retinoblastoma. However, the clinical and prognostic significance of such finding is doubtful as the lens is an avascular tissue.

\section{Statement of Ethics}

Ethical clearance was obtained.

\section{Disclosure Statement}

There is no conflict of interest with state or any other party.

\section{References}

Retinoblastoma Involving the Crystalline Lens with Spontaneous Absorption
1 Stafford WR, Yanoff M, Parnell BL: Retinoblastomas initially misdiagnosed as primary ocular inflammations. Arch Ophthalmol 1969;82:771-773.

2 Kuchynka P: Malignant epithelioma of the ciliary body. Ophthalmologica 1979;178:190193.

3 Grossniklaus HE, Zimmerman LE, Kachmer ML: Pleomorphic adenocarcinoma of the ciliary body: immunohistochemical and electron microscopic features. Ophthalmology 1990;97:763-768.
4 Gupta A, Lingam G, Krishnakumar S, Khetan $\mathrm{V}$ : Lens invasion in retinoblastoma. Pediatr Blood Cancer 2012;58:477.

5 Mal'tsev EV: Hypothetic "tumorous immunity" of the lens. Oftalmol Zh 1977;32:231.

6 Eagle RC, Spencer WH: Lens; in Spencer WH (ed): Ophthalmic Pathology, 4th edition. Philadelphia, PA: WB Saunders; 1996, pp 372-437. 